\title{
EL REGISTRO DE PERNA PERNA (LINNÉ, 1758) (MOLLUSCA: LAMELLIBRANCHIA) PARA EL ESTRECHO DE MAGALLANES ${ }^{1}$
}

\author{
THE RECORD OF PERNA PERNA (LINNÉ, 1758) (MOLLUSCA: \\ LAMELLIBRANCHIA) FROM THE MAGELLAN STRAITS ${ }^{1}$
}

Vicente Pérez ${ }^{2}$

En un trabajo de compilación taxonómica y sistemática, Osorio \& Bahamonde (1970) citan al mitílido Perna perna (Linné, 1758), como la única especie de Perna Retzius, 1788, habitante de Chile desde Concepción al estrecho de Magallanes, tomando este registro de la Décima Edición de la Zoología de Linneo (1758), cuyo "hábitat" era "Freto Magellanico" (el estrecho magallánico).

Ramorino (1978), al referirse a las especies de mitílidos cultivados en América Latina, y de importancia comercial por mayor captura, registra a Perna perna sólo para Venezuela, y analiza la cita linneana desde el punto de vista nomenclatural: Linné (1758) describió una especie de Magallanes como Mya perna; Soot-Ryen (1955) designó a Perna magellanica Retzius, 1788 = Mya perna Linné como especie tipo de Perna Retzius, atribuyéndole una distribución desde Magallanes hasta Concepción y también la costa atlántica de Sudamérica; agrega Ramorino (1978) que es muy dudosa la presencia de $P$. perna en la zona de Magallanes (localidad tipo), por lo que es posible que el nombre específico perna asignado por Linneo no se refiera a una especie de Perna o bien que el material en que basó su descripción proviniera de otra región; también menciona la opinión de Beauperthuy (1967), quien presenta la hipótesis de que Perna perna pudo haber existido en Magallanes pero pudiera haber sido desplazada ecológicamente por otros mitílidos de aguas frías.

Sin embargo, si un taxónomo, que siempre es morfólogo, examinara el habitus de Perna perna y el carácter color y el estado de carácter marrón, que lo distinguirían fácilmente de los otros mitílidos de la región de Magallanes, no quedaría ninguna duda de su ausencia. Nunca se ha sabido que un mejillón con estas características haya sido extraído de aguas australes por los mariscadores. Y el taxónomo encontraría que la mejor característica para identificarla es la cicatriz interna dividida del músculo retractor posterior (Rajagopal \& Venugopalan, 2005).

Por lo demás, el Museo Nacional de Historia Natural en su colección de moluscos chilenos no posee ningún ejemplar de dicha especie procedente de territorio nacional (Sergio Letelier,

1 Perteneciente al Proyecto Antecedentes para la Historia d las Ciencias Naturales en la región de Magallanes

2 Laboratorio de Entomología, Instituto de la Patagonia, Universidad de Magallanes, Punta Arenas. vicente.perez@umag.cl 
comunicación personal).

Para Ramorino (1978), situaciones como ésta, se explicarían por la carencia casi total de investigación taxonómica, sin aportes originales derivados de estudios reales. La revisión de la literatura malacológica muestra que ésta abunda en publicaciones reducidas a listas de especies con sus sinonimias acumuladas.

Afortunadamente el material depositado y conservado bajo estrictas normas de seguridad en diferentes museos, provenientes de expediciones y aportes de particulares, permitió que especialistas no latinoamericanos ordenaran la nomenclatura taxonómica, establecieran las sinonimias y describieran nuevas especies para la ciencia, basados en el Código Internacional de Nomenclatura Zoológica publicado por la Comisión Internacional de Nomenclatura Zoológica (1999).

Lamentablemente, la importancia de las especies de moluscos de interés comercial ha relegado a un segundo plano el estudio de los problemas taxonómicos (Ramorino, 1978), situación que en la actualidad no ha cambiado.

El objetivo de esta nota es aclarar una situación nomenclatural real de una especie reciente, ligada al estrecho de Magallanes, resaltar la importancia de los estudios taxonómicos y aportar algunos antecedentes más, extraídos de la literatura nacional y que contribuyan al conocimiento de las ciencias naturales de nuestra región.

Hupé (1854), el malacólogo de la Zoología de Claudio Gay, cita el nombre genérico Perna para nuestro país, pero sólo referido a una especie fósil (Griffin \& Nielsen, 2008); de gran utilidad para la localización de nombres científicos es la contribución de Stuardo (1960).

Philippi (1855), al recibir en el Museo Nacional de Historia Natural, numerosas conchas de moluscos procedentes de Magallanes, de parte del Gobernador Jorge Schythe, no menciona ninguna especie de Perna, y al hacer algunas comparaciones zoogeográficas de latitudes semejantes, refiriéndose a los moluscos del Cabo de Buena Esperanza (África) dice: "Toda la fauna del Cabo indica mares más calientes. Entre los Bivalvos encontramos allí los jéneros Perna..." Esta declaración es muy importante, porque él, con su gran experiencia zoológica lograda en Europa, sabía que no podría haber especies de Perna en aguas frías.

Ya no aparece Perna perna Linné, 1758, como habitante de Chile en obras recientes (Osorio, 2002), ni para la Provincia Malacológica Magallánica (Forcelli 2000), ni entre los invertebrados marinos del sur de Chile (Zagal \& Hermosilla, 2007), ni para la región de los Fiordos Chilenos (Zelaya, 2009).

Sin embargo, con Internet, invaluable sistema para obtener información por una parte, pero también fuente de incertidumbre por la facilidad con que se que pueden editar páginas en él por parte de especialistas y de los que no son tales, se ha abierto un nuevo frente de preocupación para la Taxonomía, ya que vuelven a surgir compiladores que pueden ser científicos con la suficiente eficiencia en alguna rama de la ciencia, pero que no son taxónomos. Sólo vamos a mencionar un ejemplo que puede leerse en Internet y que precisamente es de la especie que hemos comentado aquí: a 36 años desde que Ramorino (1978) analizara la nomenclatura al respecto, este año vuelve Perna perna (Linné, 1758) a anotarse entre las especies de mitílidos chilenos recientes (Aldea, 2014) lo cual introduce una errónea, infundada e innecesaria confusión en el sistema.

Y como la nomenclatura taxonómica está en permanente revisión, el género Perna Retzius parece no estar todavía libre de controversia: existe Perna Philippsson, 1788. Diss. Nova Test. Gen. 20. Y parece haberse establecido (véase la Opinión 495 del ICZN) que es Philippsson, el estudiante, y no Retzius el profesor, quien debe recibir el reconocimiento de ser el autor de la Diss. Nova Test. Gen., 1788, y de los nombres que aparecieron primero en esa obra (Vokes, 1980).

Expresamos nuestros agradecimientos al Prof. Luis Ramorino, por su gentileza en atender nuestras consultas.

\section{LITERATURA CITADA}

Acosta, V., A. Prieto Arcas \& C. Lodeiros (2006). Índice de condición de los mejillones Perna perna y Perna viridis (Bivalvia: Mytilidae) bajo un sistema suspendido de cultivo en la Ensenada de Turpialioto, Golfo de Cariaco, Venezuela. Zootecnia Tropical, 24(2):177-192. Online http:// dialnet.unirioja.es/servlet/articulo?codi 
$\mathrm{go}=2220571.13 / 06 / 2008$

Aldea V., C. (2014). Moluscos marinos de Chile. Clase Bivalvia. Fundación CEQUA. http:// www.moluscoschilenos.cl 29/09/2014

Beauperthuy, I. (1967). Los mitílidos de Venezuela (Mollusca: Bivalvia). Bol. Inst. Oceanogr.,Univ. Oriente, 6(1):7-115.

Comisión Internacional de Nomenclatura Zoológica. 1999. Código Internacional de Nomenclatura Zoológica. $4^{\mathrm{a}}$ Edición, adoptada por la Unión Internacional de Ciencias Biológicas. Versión PDF hecha disponible con la Aprobación del ITZN (2009).

Forcelli, D.O. (2000). Moluscos Magallánicos. Guía de Moluscos de Patagonia y Sur de Chile. Vásquez Mazzini Editores, Buenos Aires, Argentina.

Griffin, M. \& S.N. Nielsen. (2008). A revisión of type specimens of Tertiary molluscs from Chile and Argentina described by d'Orbigny (1842), Sowerby (1846) and Hupé (1854). Journal of Systematic Palaeontology, 6:251-316.

Hupé, L.H. (1854). Fauna Chilena. Moluscos. In: Gay. Historia Física y Política de Chile. Zoológica. 8: 1-500.

Linnaeus, C. (1758). Systema naturae per regna tria naturae, secundum clases, ordines, genera, especies, cum characteribus, differentiis, synonymis, locis. Editio decima, reformata. Laurentius Salvius: Holmiae, ii, 824 pp. Disponible online at http:///www.archive.org/details/ systemanaturae 01 linnuoft

Osorio, C. \& N. Bahamonde (1970). Lista preliminar de Lamelibranquios de Chile. Boletín del Museo Nacional de Historia Natural, 31: 185-256.

Osorio, C. 2002. Moluscos marinos en Chile. Especies de importancia económica.
Guía para su identificación. Facultad de Ciencias, Universidad de Chile. 212 pp.

Philippi, R.A. (1855). Zoología. Observaciones sobre las conchas de Magallanes. Anales de la Universidad de Chile, 0:203-213.

Rajagopal, S. \& V.P. Venugopalan (2005). Ecology of Perna perna. Global Invasive Species Database http//www.issg.org/database/ species /ecology.asp? $\mathrm{si}=742 \& \mathrm{fr}=1 \&$ sts $16 / 06 / 2008$

Ramorino, L. [1978]". SR/1. Biología de Moluscos cultivados en América Latina. La acuicultura en América Latina. Documentos de Reseña, 2:1-5. Online http:/www.fao. org/docrep/005/ac867s/ac867s01.htm 12/06/2008.

Soot-Ryen, T. (1955). A report on the family Mytilidae (Pelecypoda). Rep. Allan Hancock Pac. Exp., 20(1):1-174.

Stuardo, C. (1960). Historia Física y Política de Chile de don Claudio Gay. Índices alfabéticos de la Botánica y de la Zoología. Publicaciones del Centro de Estudios Entomológicos, Facultad de Filosofía y Educación, Universidad de Chile, 3:297359.

Vokes, H.E. (1980). Genera of the Bivalvia: A Systematic and Bibliographic Catalogue (Revised and Updated). Paleontological Research Institution.

Zagal, C.A. \& C. Hermosilla (2007). Guía de invertebrados marinos del sur de Chile. Fantástico Sur Birding Ltda. Punta Arenas. Chile.

Zelaya, D.G. (2009). Bivalvia - Bivalvos. In Häussermann, V. \& G. Försterra (Eds.), Fauna Marina Bentónica de la Patagonia Chilena. Santiago. Nature in Focus, pp. 426-454.

* El año de publicación nos fue proporcionado por el Prof. Luis Ramorino. 
